\title{
Paraoxonase (PON1) L55M and 0192R polymorphisms in major depression and bipolar affective disorder
}

\author{
1 Department of Psychiatry, Faculty of Medicine, Gaziosmanpasa University, Tokat, Turkey. \\ 2 Department of Clinical Psychology, Institute of Social Sciences, Isik University, Istanbul, Turkey. \\ ${ }^{3}$ Department of Medical Biology, Faculty of Medicine, Gaziosmanpaşa University, Tokat, Turkey. \\ ${ }_{4}$ Psychiatry Department, Haydarpasa Numune Training and Research Hospital, Istanbul, Turkey. \\ ${ }^{5}$ Department of Biochemistry, Faculty of Medicine, Gaziosmanpaşa University, Tokat, Turkey \\ ${ }^{6}$ Department of Biostatistics, School of Medicine, Gaziosmanpasa University, Tokat, Turkey. \\ ${ }^{*}$ At Gaziosmanpaşa University when the study was conducted.
}

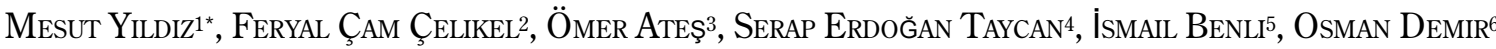

Received: $12 / 15 / 2016$ - Accepted: 4/3/2017

DOI: 10.1590/0101-60830000000123

\begin{abstract}
Background: Oxidative and nitrosative stress pathways, along with immune-inflammatory response, might play an important role in the pathogenic mechanisms underlying major depression and bipolar disorder. Objective: The aim of the present study is to investigate paraoxonase 1 polymorphisms and its correlations with disease parameters in patients with major depression and bipolar affective disorder. Methods: PON1 L55M and Q192R single nucleotide polymorphisms were analyzed in a group consisted of 100 patients with major depression, and 100 patients with bipolar affective disorder and 96 healthy controls. Polymorphisms were analyzed by using polymerase chain reaction. Results: Our findings reported no association between Q192R and L55M polymorphisms of PON1 and major depression and bipolar disorder. Additionally, there was no association between the PON1 genotypes and disease variables in both depressed and bipolar patients. Discussion: Evaluating the different stages of patients with affective disorders and and investigating the connection between PON1 polymorphisms and treatment outcomes will help us to clarify the relationship between PON1 and mood disorders.
\end{abstract}

Yildiz M et al. / Arch Clin Psychiatry. 2017;44(3):73-6

Keywords: Major depression, bipolar affective disorder, paraoxonase (PON1), polymorphism, association study.

\section{Introduction}

Mood disorders are thought to be caused by a combination of environmental, psychological, biological and genetic factors. It was shown that oxidative and nitrosative stress pathways, along with immune-inflammatory response, might play an important role in the pathogenic mechanisms underlying major depression and bipolar disorder ${ }^{1}$. Reactive oxygen species affect both the immune-inflammatory pathways and the expression of key neurotransmitters which are involved in the pathophysiology of depression ${ }^{2}$. Studies demonstrated discrepancies in antioxidant enzyme levels in different stages of bipolar affective disorder ${ }^{3,4}$. Additionally, adding antioxidant agents to treatment of patients with bipolar affective disorder caused a substantial decrease in depressive symptoms and and increase in clinical functioning or quality of life measures ${ }^{5}$.

Paraoxonase is a calcium-dependent esterase that catalyzes the hydrolysis of neurotoxins such as organophosphates and aromatic carboxylic acids ${ }^{6}$. The paraoxonase (PON) gene family has three members, PON1, PON2, andPON3. Human paraoxonase 1 (PON1) is a high-density lipoprotein (HDL)-associated serum enzyme that exhibits a broad substrate specificity? The PON1 enzyme has both paraoxonase and arylesterase activity and show antiinflammatory and antioxidative properties $^{8}$. The PON1 gene contains two common polymorphisms found in the PON1 coding region, leading to a glutamine (Q) $\rightarrow$ arginine (R) substitution at position 192 (Q192R; rs 662) and to a leucine $(\mathrm{L}) \rightarrow$ methionine $(\mathrm{M})$ substitution at position 55 (L55M; rs $854560)^{9}$. The L55M polymorphism affects the enzyme concentration and the Q192R polymorphism is responsible for the hydrolytic activity of the enzyme ${ }^{10,11}$. Studies concerning the relationship between PON1 polymorphism and mood disorders exhibited inconsistent results.
It was reported that PON1 Q192R polymorphism may be associated with symptoms of depression in older women ${ }^{12}$. Another study demonstrated that PON1 Q192R-smoking interaction predicted the odds of depression ${ }^{13}$. Contrarily, no associations were detected between mood disorders and any of the Q192R genotypes ${ }^{14}$. In a genome-wide association study, thew authors reported no associations between major depression and the Q192R polymorphism or any other polymorphism in the PON1 gene ${ }^{15}$. Also, no significant association was found between PON1Q192R polymorphism and depression in populationbased studies ${ }^{16}$. While some studies found no differences in terms of PON1 activity between patients with depression and controls ${ }^{17}$; others demonstrated a diminished PON1 activity ${ }^{13,18,19}$. Furthermore, it was indicated that antidepressant treatment increased the lowered paraoxonase/arylesterase levels ${ }^{19}$.

It was demonstrated that carrying homozygote or heterozygote mutated alleles of L55M and Q192R might cause susceptibility to bipolar I disorder ${ }^{9}$. The odds of bipolar disorder were increased by the QQ genotype of Q192R in smokers ${ }^{13}$. In a genome-wide association study, no associations between bipolar disorder and the Q192R polymorphism or any other polymorphism in the PON1 gene was detected. Two studies investigated PON1 activity in bipolar patients. The first one presented normal PON1 activity but the other showed decreased PON1 activity in bipolar patients ${ }^{13,20}$. In a study consisting of both depressed and bipolar patients; lowered PON1 activity was found to be associated with comorbid mood disorders and tobacco use disorder ${ }^{14}$. In terms of polymorphisms, there were no significant associations between the patient groups and any of the three PON1 Q192R genotypes in the same study.

The aim of the present study is to investigate paraoxonase 1 polymorphisms and its correlations with disease parameters in patients with major depression and bipolar affective disorder. 


\section{Methods}

This study used the DNA specimens of a former study entitled: "Investigation of Dopamine- $\beta$-hydroxylase Polymorphism in Patients with Major Depression, Bipolar Affective Disorder and Schizophrenia". The study group consisted of 100 patients with major depression (83 females and 17 males), and 100 patients with bipolar affective disorder ( 49 females and 51 males) and 96 healthy controls (48 females and 48 males). All depressed and bipolar patients were followed up at the Outpatient Clinic of the Psychiatry Department of Gaziosmanpasa University, School of Medicine. Diagnoses of major depression and bipolar disorder were made according to the DSM-IV (Diagnostic and Statistical Manual-IV) criteria (American Psychiatric Association, 1994)21. The control subjects were recruited from hospital staff. The study protocol was approved by the Clinical Researches Ethics Committee, and the written informed consents were obtained from the study participants.

\section{Genotyping}

Genomic DNA was extracted from peripheral leukocytes from EDTA-anticoagulated blood using the High Pure Polymerase Chain Reaction Template Preparation Kit (Roche Molecular Biochemicals, Mannheim, Germany) according to the manufacturer's instructions. To identify PON1 L55M and Q192R singlenucleotide polymorphisms (SNPs), genotyping was performed using commercially synthesized primers and fluorescently labeled probes (Metabion, Martinsried, Germany) and the LightCycler 480 II Real-Time Polymerase Chain Reaction System (Roche Diagnostics). The genotyping method was based on methods developed previously for genotyping both PON1 55 and 192 polymorphisms using LightCycler realtime polymerase chain reaction technology, which relies on fluorescence resonance energy transfer ${ }^{22}$. Target fragments of the human PON1 gene were amplified with specific primers. To detect the PON1 L55M polymorphism, $10 \mathrm{pmol}$ of the forward primer 5'-CCTGCAATAATATGAAACAACCTG-3' and the reverse primer 5'-CTAGAACACAGAAAAGTGAAAGAAAAC-3' and 3 pmol of the sensor probe 5'-CTCTGAAGACATGGAGATACTGCCfluorescein-3' and the anchor probe 5'-LCRed640-ATGGACTGG CTTTCATTAGCTCTGTGAGT-3' were added to genomic DNA. To detect the PON1 Q192R polymorphism,we also used $10 \mathrm{pmol}$ of the forward primer 5'-ATTGTTGCTGTGGGACCTGAG-3' and the reverse primer 5'-CCTTCTGCCACCACTCGAAC-3' and 3 pmol of the sensor probe 5'-CCCCTACTTACAATCCTGGGAGATfluorescein-3' and the anchor probe 5'-LCRed705ATTTGGGTTTAGCGTGGTCGTATGTTG-3'. Melting curves were transformed to melting peaks by plotting the negative derivative of the fluorescence signal versus the temperature. The genotypes were identified by creating a melting curve with specific melting points.

\section{Statistical analysis}

Descriptive analyses were performed to provide information on general characteristics of the study population. One way ANOVA test was used to compare the continuous data among groups. The continuous data were presented as the mean \pm standard deviation. Chi-Square test was used to compare the categorical data between/ among groups. Categorical variables were presented as a count and percentage. A p-value $<0.05$ was considered significant. Analyses were performed using SPSS 19 (IBM SPSS Statistics 19, SPSS inc., an IBM Co., Somers, NY) ${ }^{23}$.

\section{Results}

The baseline clinical and demographics features of patients with major depression and bipolar disorder are shown in Table 1 and
Table 2. The mean age of the depressed group, bipolar group and controls were $38.21 \pm 12.07,41.19 \pm 12.25$ and $37.34 \pm 10.21$ respectively. No significant difference in the mean ages and gender was observed between the bipolar patient group and the control group. The mean age was not different between depressed group and controls but the depressed group was different from controls in terms of gender. Namely, the the majority of the depressed group consisted of females. All the PON1 gene polymorphisms analyzed were in Hardy-Weinberg equilibrium. Allele and genotype frequencies were not different between patients with depression and controls in with regard to L55M and Q192R polymorphisms of PON1 (Table 3). Likewise, there was not a significant difference of to L55M and Q192R polymorphism between patients with bipolar disorder and controls (Table 4). Additionally, there was no association between the PON1 genotypes and disease variables in both depressed and bipolar patients.

Table 1. Baseline clinical and demographics features of the 100 study patients with major depression

\begin{tabular}{|l|c|}
\hline Characteristic & Depressed patients group \\
\hline Gender, no. male/female (male \%/female \%) & $13 / 87(13.0 / 87.0)$ \\
\hline Age, mean \pm SD (range) years & $38.21 \pm 12.07$ \\
\hline
\end{tabular}

Table 2. Baseline clinical and demographics features of the 100 study patients with bipolar disorder

\begin{tabular}{|l|c|}
\hline Characteristic & Bipolar patients group \\
\hline Gender, no. male/female (male \%/female \%) & $49 / 51(49.0 / 51.0)$ \\
\hline Age, mean \pm SD (range) years & $41.18 \pm 12.27$ \\
\hline Age of onset, mean \pm SD (range) years & $25.21 \pm 9.86$ \\
\hline Bipolar disorder subtype, $\mathrm{n}(\%)$ & \\
\hline Type 1 & $87(87 \%)$ \\
\hline Type 2 & $13(13 \%)$ \\
\hline Rapid cycling, $\mathrm{n}(\%)$ & $17(17 \%)$ \\
\hline Seasonal pattern, $\mathrm{n}(\%)$ & $59(59 \%)$ \\
\hline Alcohol/drug use, $\mathrm{n}(\%)$ & $10(10 \%)$ \\
\hline Suicidal behavior, $\mathrm{n}(\%)$ & $26(26 \%)$ \\
\hline Psychotic feature, $\mathrm{n}(\%)$ & $55(55 \%)$ \\
\hline Hospitalization, $\mathrm{n}(\%)$ & $69(69 \%)$ \\
\hline Family history of BPD, $\mathrm{n}(\%)$ & $33(33 \%)$ \\
\hline Family history of suicide, $\mathrm{n}(\%)$ & $13(13 \%)$ \\
\hline
\end{tabular}

Table 3. PON1 polymorphisms in patients with major depression and healthy controls

\begin{tabular}{|l|c|c|c|}
\hline PON locus & $\begin{array}{c}\text { Healthy controls } \\
n=96(\%)\end{array}$ & $\begin{array}{c}\text { Major depression } \\
\text { patients } \\
n=100(\%)\end{array}$ & $P$ \\
\hline PON55L/M & $40(41.7)$ & $37(37.0)$ & 0.56 \\
L/L & $42(43.7)$ & $43(43.0)$ & \\
L/M & $14(14.6)$ & $20(20.0)$ & 0.15 \\
M/M & $122(63.5)$ & $117(58.5)$ & \\
Alleles & $70(36.5)$ & $83(41.5)$ & \\
L & & & 0.61 \\
M & $43(44.8)$ & $47(47.0)$ & \\
\hline PON1920/R & $39(40.6)$ & $43(43.0)$ & \\
Q/0 & $14(14.6)$ & $10(10.0)$ & 0.24 \\
Q/R & $125(65.0)$ & $137(68.5)$ & \\
R/R & $67(35.0)$ & $63(31.5)$ & \\
Alleles & \multicolumn{2}{|}{} \\
O & \multicolumn{2}{|l}{} \\
R & & \\
\hline
\end{tabular}


Table 4. PON1 polymorphisms in patients with bipolar affective disorders and healthy controls

\begin{tabular}{|l|c|c|c|}
\hline PON locus & $\begin{array}{c}\text { Healthy controls } \\
n=96(\%)\end{array}$ & $\begin{array}{c}\text { Bipolar patients } \\
n=100(\%)\end{array}$ & $P$ \\
\hline PON55L/M & $40(41.7)$ & $39(39.0)$ & 0.89 \\
L/L & $42(43.7)$ & $47(47.0)$ & \\
L/M & $14(14.6)$ & $14(14.0)$ & 0.41 \\
M/M & $122(63.5)$ & $125(62.5)$ & \\
Alleles & $70(36.5)$ & $75(37.5)$ & \\
L & & & \\
M & $43(44.8)$ & $44(44.0)$ & 0.95 \\
\hline PON1920/R & $39(40.6)$ & $40(40.0)$ & \\
Q/0 & $14(14.6)$ & $16(16.0)$ & 0.41 \\
Q/R & & & \\
R/R & $125(65.0)$ & $128(64.0)$ & \\
Alleles & $67(35.0)$ & $72(36.0)$ & \\
O & &
\end{tabular}

\section{Discussion}

The present study examined the L55M and Q192R polymorphisms of PON1 gene in patients with depression and bipolar disorder. We did not find any association between the L55M and Q192R polymorphisms and major depression and bipolar disorder. Also, no association was found between the PON1 genotypes and disease variables in both patient groups.

The lack of association between the L55M and Q192R polymorphisms and major depression in the present study is in line with Nunes et al's report ${ }^{14}$. Although, they did not distinguish depressed and bipolar patients and they only looked for the Q192R polymorphisms but not for the L55M polymorphisms; but they found no association between patients with mood disorders and any of the three PON1 Q192R genotypes. In a similar manner, no associations between major depression and the Q192R polymorphism or any other polymorphism in the PON1 gene have been found in a genomewide association study ${ }^{15}$. However, there are some contradictory findings. In a study exploring the PON Q192R polymorphism in major depression in relation to nicotine dependence, it was demonstrated that PON Q192R-smoking interaction might play a role in depression ${ }^{13}$. A different study including British women aged 60-79 years, R allele of PON1 Q192R was found to be associated with increased odds of depression ${ }^{12}$. Large differences between ethnic populations are known in the PON1 genotype distribution ${ }^{24}$. So, it may be the reason for differences among studies. The majority of the subjects in the depressed group were female and it was significantly different from controls. As it was shown that gender had no impact on PON1 polymorphism ${ }^{25}$, it was thought that the gender difference in the present study did not confound the results. The contradictive results are also present for PON1 activity. No differences were found in terms of PON1 activity between patients with depression and controls $^{17}$. A diminished PON1 activity was also reported ${ }^{13,18,19}$. In addition, long-term $\mathrm{AD}$ treatment seems to increase the paraoxonase/ arylesterase levels in patients with depression ${ }^{19}$. In the present study, we did not measure PON1 activity. As in our study, the vast majority of the studies in the literature did not include any measure of PON1 activity.

We did not find any association between any association between the L55M and Q192R polymorphisms and bipolar disorder. Ezzaher et al. demonstrated that carrying homozygote or heterozygote mutated alleles of L55M and Q192R might cause susceptibility to bipolar I disorder9. It was indicated that the QQ genotype of Q192R in smokers increased the risk of bipolar disorder ${ }^{13}$. Our findings are consistent with a genome-wide association study reporting no associations between bipolar disorder and the Q192R polymorphism or any other polymorphism in the PON1 gene ${ }^{26}$. In terms of PON1 activity in bipolar patients; one study demonstrated normal activity ${ }^{13}$ but the other showed decreased PON1 activity ${ }^{20}$. The results of the present study and the discrepancies between the other studies might be associated with the distinct genotypic distribution of PON1 across different ethnic populations.

We also found no association between clinical variables of depression and bipolar disorder and the L55M and Q192R polymorphismS of PON1. Diminished serum paraoxonase and arylesterase activities and polymorphisms of PON1 in humans have been have been linked to heightened systemic oxidative stres ${ }^{8,27}$. So, it could be expected that, the more serious forms of mood disorders might be associated with lower levels or lower activity of antioxidant properties.

Polymorphism regarding PON1 have not been studied too much in other psychiatric disorders. In a study including patients with schizophrenia, their relatives and healthy controls; authors suggested that the subjects carrying R allele or RR genotype of Q192R polymorphism might be susceptible to schizophrenia and subjects with QQ or LL of L55M polymorphism might be protected against schizophrenia ${ }^{28}$. Another study looked for an association between Q192R and L55M polymorphisms of PON1 and autism spectrum disorders, but they found no association ${ }^{29}$.

The results of the present study must be interpreted within the limitations of the study. Firstly, larger sample size of groups would be beneficial and the sample of our depressed and bipolar patients may not be representative of the whole patient populations. Secondly, our work is a cross sectional study that does not permit to follow-up of biological parameters. We did not measure PON activity but it is the common limitation of such association studies. Another limitation of the study is that reports from association studies constitute tentative knowledge and must be interpreted with caution ${ }^{30}$.

In conclusion, our findings reported no association between Q192R and L55M polymorphisms of PON1 and major depression and bipolar disorder, suggesting that this polymorphism might not play a role in the physiopathology of mood disorders. There were also no significant associations between the polymorphisms of the PON1 gene and the clinical and the demographic characteristics of patients. Prospective studies with larger sample sizes evaluating the different stages of patients investigating the connection between PON1 polymorphisms and treatment outcomes will help us to clarify the relationship between PON1 and mood disorders.

\section{Acknowledgments}

This study was supported by the Gaziosmanpasa University (project no: 2015/28). The authors would like to thank the patients and the controls for their participation.

\section{Conflict of interest}

The authors do not have an affiliation with or financial interest in any organization that might pose a conflict of interest that are directly relevant to the contents of the paper.

\section{References}

1. Siwek M, Sowa-Kućma M, Dudek D, Styczeń K, Szewczyk B, Kotarska $\mathrm{K}$, et al. Oxidative stress markers in affective disorders. Pharmacol Rep. 2013;65(6):1558-71.

2. Moylan S, Berk M, Dean OM, Samuni Y, Williams LJ, O’Neil A, et al. Oxidative \& nitrosative stress in depression: why so much stress? Neurosci Biobehav Rev. 2014;45:46-62.

3. Andreazza AC, Cassini C, Rosa AR, Leite MC, Almeida LM, Nardin P, et al. Serum S100B and antioxidant enzymes in bipolar patients. J Psychiatr Res. 2007;41(6):523-9.

4. Machado-Vieira R, Dietrich MO, Leke R, Cereser VH, Zanatto V, Kapczinski F, et al. Decreased plasma brain derived neurotrophic factor levels in unmedicated bipolar patients during manic episode. Biol Psychiatry. 2007;61(2):142-4.

5. Berk M, Copolov DL, Dean O, Lu K, Jeavons S, Shapkaitz I, et al. Antioxidant treatment of the glutathione deficiency in bipolar disorder with 
$\mathrm{N}$-acetylcysteine: a double-blind randomised placebo controlled trial. Bipolar Disord. 2007;9:8-9.

6. La Du BN, Aviram M, Billecke S, Navab M, Primo-Parmo S, Sorenson $\mathrm{RC}$, et al. On the physiological role(s) of the paraoxonases. Chem Biol Interact. 1999;119-120:379-88

7. Furlong CE, Suzuki SM, Stevens RC, Marsillach J, Richtera RJ, Jarvika GP, et al. Human PON1, a biomarker of risk of disease and exposure. Chem Biol Interact. 2010;187(1-3):355-61.

8. Primo-Parmo SL, Sorenson RC, Teiber J, La Du BN. The human serum paraoxonase/arylesterase gene (PON1) is one member of a multigene family. Genomics. 1996;33(3):498-507.

9. Ezzaher A, Mouhamed DH, Mechri A, Neffati F, Rejeb J, Omezzine A, et al. Association between bipolar I disorder and the L55M and Q192R polymorphisms of the paraoxonase 1 (PON1) gene. J Affect Disord. 2012;139(1):12-7.

10. Adkins S, Gan KN, Mody M, La Du BN. Molecular basis for the polymorphic forms of human serum paraoxonase/arylesterase: glutamine or arginine at position 191, for the respective A or B allozymes. Am J Hum Genet. 1993;52(3):598-608.

11. Goswami B, Tayal D, Gupta N, Mallika V. Paraoxonase: a multifaceted biomolecule. Clin Chim Acta. 2009;410(1-2):1-12.

12. Lawlor DA, Day IN, Gaunt TR, Hinks LJ, Timpson N, Ebrahim S, et al. The association of the paraoxonase (PON1) Q192R polymorphism with depression in older women: findings from the British Women's Heart and Health Study. J Epidemiol Community Health. 2007;61(1):85-7.

13. Bortolasci CC, Vargas HO, Souza-Nogueira A, Barbosa DS, Moreira EG, Nunes SOV, et al. Lowered plasma paraoxonase (PON) 1 activity is a trait marker of major depression and PON1 Q192R gene polymorphismsmoking interactions differentially predict the odds of major depression and bipolar disorder. J Affect Disord. 2014;159:23-30.

14. Vargas Nunes SO, Pizzo de Castro MR, Moreira EG, Guembarovski RL, Barbosa DS, Vargas HO, et al. Association of paraoxonase (PON)1 activity, glutathione S-transferase GST T1/M1 and STin.2 polymorphisms with comorbidity of tobacco use disorder and mood disorders. Neurosci Lett. 2015;585:132-7.

15. Sullivan PF, de Geus EJ, Willemsen G, James MR, Smit JH, Zandbelt T, et al. Genome-wide association for major depressive disorder: a possible role for the presynaptic protein piccolo. Mol Psychiatry. 2009;14(4):359-75.

16. Rice NE, Bandinelli S, Corsi AM, Ferrucci L, Guralnik JM, Miller MA, et al. The paraoxonase (PON1) Q192R polymorphism is not associated with poor health status or depression in the ELSA or INCHIANTI studies. Int J Epidemiol. 2009;38(5):1374-9.
17. Sarandol A, Sarandol E, Eker SS, Karaagac EU, Hizli BZ, Dirican M, et al. Oxidation of apolipoprotein B-containing lipoproteins and serum paraoxonase/arylesterase activities in major depressive disorder. Prog Neuropsychopharmacol Biol Psychiatry. 2006;30(6):1103-8.

18. Barim AO, Aydin S, Colak R, Dag E, Deniz O, Sahin I. Ghrelin, paraoxonase and arylesterase levels in depressive patients before and after citalopram treatment. Clin Biochem. 2009;42(10-11):1076-81.

19. Kotan VO, Sarandol E, Kirhan E, Ozkaya G, Kirli S. Effects of long-term antidepressant treatment on oxidative status in major depressive disorder: a 24-week follow-up study. Prog Neuropsychopharmacol Biol Psychiatry. 2011;35(5):1284-90.

20. Ezzaher A, Mouhamed DH, Mechri A, Araoud M, Neffati F, Douki W, et al. Lower paraoxonase 1 activity in Tunisian bipolar I patients. Ann Gen Psychiatry. 2010;9:36.

21. American Psychiatric Association. Diagnostic and Statistical Manual of Mental Disorders: DSM-IV. 4th ed. Washington (DC): American Psychiatric Association; 1994.

22. Pocsai Z, Tóth Z, Paragh G, Szeles G, Adany R. Rapid genotyping of paraoxonase 55 and 192 mutations by melting point analysis using real time PCR technology. Clin Chim Acta. 2003;(1-2)332:31-6.

23. IBM Corp. Released 2010. IBM SPSS Statistics for Windows, Version 19.0. Armonk, NY: IBM Corp.

24. Koda Y, Tachida H, Soejima M, Takenaka O, Kimura H. Population differences in DNA sequence variation and linkage disequilibrium at the PON1 gene. Ann Hum Genet. 2004;68:110-9.

25. Rojas-Garcia AE, Solis-Heredia MJ, Pina-Guzman B, Vega L, Lopez-Carrillo L, Quintanilla-Vega B. Genetic polymorphisms and activity of PON1 in a Mexican population. Toxicol Appl Pharmacol. 2005;205(3):282-9.

26. Ferreira MA, O’Donovan MC, Meng YA, Jones IR, Ruderfer DM, Jones L, et al. Collaborative genome-wide association analysis supports a role for ANK3 and CACNA1C in bipolar disorder. Nat Genet. 2008;40(9):1056-8.

27. Tang WW, Hartiala J, Fan Y, Wu Y, Stewart AF, Erdmann J, et al. Clinical and genetic association of serum paraoxonase and arylesterase activities with cardiovascular risk. Arterioscler Thromb Vasc Biol. 2012;32(11):2803-12.

28. Kucukali CI, Aydin M, Ozkok E, Orhan N, Cakir U, Kilic G, et al. Paraoxonase-1 55/192 genotypes in schizophrenic patients and their relatives in Turkish population. Psychiatr Genet. 2008;18(6):289-94.

29. Paşca SP, Nemeş B, Vlase L, Gagyi CE, Dronca E, Miu AC, et al. High levels of homocysteine and low serum paraoxonase 1 arylesterase activity in children with autism. Life Sci. 2006;78(19):2244-8.

30. Sullivan PF. Spurious genetic associations. Biol Psychiatry. 2007;61(10):1121-6. 